\title{
Inhibition of Notch signalling ameliorates experimental inflammatory arthritis
}

\author{
Jong-Sung Park, ${ }_{1}$ Seol-Hee Kim, ${ }^{1,2}$ Kwangmeyung Kim, ${ }^{3}$ Cheng-Hao Jin, ${ }^{4}$ \\ Ki Young Choi, ${ }_{1}^{5}$ Jiyeon Jang, ${ }^{1}$ Yuri Choi, ${ }^{1}$ A-Ryeong Gwon, ${ }^{1}$ Sang-Ha Baik, ${ }_{1}^{1}$ \\ Ui Jeong Yun, ${ }^{1}$ Su Young Chae, ${ }^{1}$ Seulki Lee, ${ }^{6}$ Young Mo Kang, ${ }^{7}$ Kang Choon Lee, ${ }^{1}$ \\ Thiruma V Arumugam, ${ }^{1,8}$ Mark P Mattson, ${ }^{9,10}$ Jae Hyung Park, ${ }^{2}$ Dong-Gyu Jo ${ }^{1}$
}

\begin{abstract}
Handling editor Tore K Kvien
- Additional material is

published online only. To view please visit the journal online (http://dx.doi.org/10.1136/ annrheumdis-2013-203467).

For numbered affiliations see end of article.

\section{Correspondence to Professor Dong-Gyu Jo, School of Pharmacy, Sungkyunkwan University, 300 Cheoncheon-Dong, Suwon 440-746, Korea; jodg@skku.edu, or Professor Jae Hyung Park, Departments of Polymer Science and Chemical Engineering, Sungkyunkwan University, 300 Cheoncheon- Dong, Suwon 440-746, Korea; jhpark1@skku.edu}

JSP, SHK and KK contributed equally.

Received 15 February 2013 Revised 9 August 2013 Accepted 26 October 2013 Published Online First 19 November 2013 \begin{tabular}{l} 
To cite: Park J-S, Kim S-H, \\
Kim K, et al. Ann Rheum Dis \\
2015;74:267-274. \\
\hline
\end{tabular}

\section{ABSTRACT}

Objective To test the hypothesis that Notch signalling plays a role in the pathogenesis of rheumatoid arthritis (RA) and to determine whether pharmacological inhibition of Notch signalling with $\gamma$-secretase inhibitors can ameliorate the RA disease process in an animal model.

Methods Collagen-induced arthritis was induced in C57BL/6 or Notch antisense transgenic mice by immunisation with chicken type II collagen (CII). C57BL/ 6 mice were administered with different doses of inhibitors of $\gamma$-secretase, an enzyme required for Notch activation, at disease onset or after onset of symptoms. Severity of arthritis was monitored by clinical and histological scores, and in vivo non-invasive near-infrared fluorescence (NIRF) images. Micro-CT was used to confirm joint destruction. The levels of $\mathrm{Cll}$ antibodies and cytokines in serum were determined by ELISA and bead-based cytokine assay. The expression levels of cytokines were studied by quantitative PCR in rheumatoid synovial fibroblasts.

Results The data show that Notch signalling stimulates synoviocytes and accelerates their production of proinflammatory cytokines and immune responses involving the upregulation of $\lg \mathrm{G} 1$ and $\lg \mathrm{G} 2 \mathrm{a}$.

Pharmacological inhibition of $\gamma$-secretase and antisensemediated knockdown of Notch attenuates the severity of inflammatory arthritis, including arthritis indices, paw thickness, tissue damage and neutrophil infiltration, and reduces the levels of active NF- $\mathrm{KB}$, ICAM-1, proinflammatory cytokines and matrix metalloproteinase3 activity in the mouse model of RA.

Conclusions These results suggest that Notch is involved in the pathogenesis of RA and that inhibition of Notch signalling is a novel approach for treating RA.

\section{INTRODUCTION}

The Notch-1 (Notch) signalling pathway regulates cell development, differentiation, proliferation, survival and apoptosis. ${ }^{1}{ }^{2}$ In mammalian cells, there are four Notch receptors (Notch-1 through Notch-4) and five Notch ligands (Delta-like [DLL]-1/-3/-4 and Jagged-1/-2), which are all transmembrane proteins. Binding of a ligand to Notch initiates a two-step proteolytic cleavage of Notch, first by an extracellular ADAM family protease, and then by the intramembrane $\gamma$-secretase complex that releases Notch intracellular domain (NICD). ${ }^{3} 4$ NICD then translocates into the nucleus, where it forms a transcriptional activator complex with the CSL family of transcription factors (C-promoter binding factor $1 /$ recombination signal sequence binding protein $\mathrm{J} \kappa$, suppressor of hairless, and Lag-1) and modulates the expressions of target genes such as Hes family of transcription factors members and $p 21 .^{1}$

Rheumatoid arthritis (RA) is a complex chronic, progressive inflammatory disease involving hyperplasia of synovial tissues and destruction of joint architecture such as cartilage, bone and ligaments. ${ }^{5}$ Data support the notion that Notch signalling is involved in the pathogenesis of RA. The expression and activation of Notch occur in osteoarthritis cartilage and RA synoviocytes. ${ }^{7-10}$ Notch signalling is involved in the TNF $\alpha$-induced proliferation of RA synoviocytes, ${ }^{10}$ and Jagged-1 can modulate collagen-induced arthritis (CIA) progression by affecting CD8 ${ }^{+}$T cell responses. ${ }^{11} \mathrm{~T}$ helper (Th) cells from RA patients exhibit an altered expression profile of Notch receptors and enhanced activation of Notch signalling. ${ }^{12}$ Recently, the interactions between the Notch signalling pathway and angiogenesis have been described in RA. Notch signalling mediates hypoxia and vascular endothelial growth factor (VEGF)/angiopoietin2 (Ang2)-induced angiogenesis in RA. ${ }^{13}{ }^{14}$ Notch signalling is also involved in the inflammatory responses of multiple sclerosis, glomerular disease and stroke. ${ }^{15-19}$ The amount of NICD is increased in diabetic nephropathy and focal segmental glomerulosclerosis. ${ }^{16}$ In addition, Notch signalling contributes to neuronal death after cerebral ischaemia by enhancing apoptotic cascades in neurons and by microglia-mediated inflammatory response. ${ }^{17-19}$

Although the functional involvement of the Notch signalling in severe inflammatory diseases has been previously suggested, Notch signalling has not been established as a therapeutic target for RA. Moreover, the mechanisms by which Notch inhibition might slow or stop the progression of RA are unknown. Here we report that suppression of Notch signalling using antisense technology and low-molecular-weight $\gamma$-secretase inhibitors ameliorate the disease process in a mouse model of RA.

\section{MATERIALS AND METHODS}

Detailed materials and methods can be found in the online supplementary data. 


\section{Mice}

Mice overexpressing Notch antisense (NAS) under the control of a mouse mammary tumour virus long terminal repeat promoter were generated, backcrossed to C57BL/6 mice and characterised as previously described. ${ }^{20}$ This study was reviewed and approved by the Institutional Animal Care and Use Committee (IACUC) of Sungkyunkwan University School of Medicine (SUSM).

\section{Collagen-induced arthritis}

CIA was induced in C57BL/6 or NAS mice as previously described. $^{21}$

\section{Synoviocyte culture}

Fibroblast-like synoviocytes were isolated from synovial tissues obtained during joint replacement surgery from patients with RA, defined according to the criteria of American College of Rheumatology. ${ }^{22}$

\section{Non-invasive near-infrared fluorescence imaging}

For optical imaging of inflammation in arthritic joints, hydrophobically modified glycol chitosan (HGC) nanoparticles were used. HGC nanoparticles were prepared by conjugating glycol chitosan with hydrophobic $5 \beta$-cholanic acid in the presence of 1-ethyl-3-(3-dimethylaminopropyl)-carbodiimide (EDAC) and $\mathrm{N}$-hydroxysuccinimide (NHS), as previously described. ${ }^{23}$

\section{Immunoblotting}

Briefly, $20 \mu \mathrm{g}$ of proteins were separated by SDS-PAGE and transferred to polyvinlylidene fluoride (PVDF) membranes. Membranes were blocked in 5\% non-fat milk for $1 \mathrm{~h}$ at room temperature and incubated overnight at $4{ }^{\circ} \mathrm{C}$ and analysed with antibodies raised against Notch-1 (upstate, Lake Placid, New York, USA), activated Notch-1 (NICD) (Abcam, Cambridge, UK), Hes-1 (Santacruz, Santa Cruz, California, USA), ICAM-1 (Santacruz), phospho-p65 (Cell Signaling, Danvers, Massachusetts, USA) or GAPDH (Thermo, Walteham, Washington, USA).

\section{Micro-CT}

At 35 days after first immunisation of CII, CT images of the knee joints and hind limbs of control and CIA mice were obtained using a micro-CT (Skyscan 1076; SKYSCAN N.V., Kontich, Belgium).

\section{Statistical analysis}

Prism V.5.0 (Graphpad Software, San Diego, California, USA) was used for the analysis. One-way analysis of variance was used for multiple group analysis. The two-tailed unpaired t test was used to analyse data from two groups. p Values of less than 0.05 were considered significant.

\section{RESULTS}

\section{A $\gamma$-secretase inhibitor reduces the severity of $\mathrm{CIA}$}

We first determined whether a $\gamma$-secretase inhibitor would impact the disease process in a mouse model of CIA that show many clinical and histopathological features of RA. ${ }^{24}$ To avoid researcher bias, the experimenter who scored the clinical condition of the mice and the histological sections for disease was unaware of the treatment history of the mice. We found that administering $10 \mathrm{mg} / \mathrm{kg}$ or $20 \mathrm{mg} / \mathrm{kg}$ of the $\gamma$-secretase inhibitor DAPT (N-[N-(3,5-difluorophenacetyl-1-alanyl)]-S-phenyl-glycine t-butyl ester) starting at 21 days after primary immunisation significantly and dose-dependently improved clinical outcomes, including arthritis indices and paw thickness of CIA, compared with the vehicle control group (figure 1A,B). Histologically, the vehicle-treated CIA mice showed extensive synovitis, neutrophil infiltration, destruction of articular cartilage and bone erosion. In contrast, the joints of DAPT-treated CIA mice showed significantly reduced synovitis, cartilage destruction, bone erosion and neutrophil infiltration (figure 1C,D).

\section{In vivo imaging and analysis of arthritis progression in $\gamma$-secretase inhibitor-treated CIA mice}

The accumulation of nanoparticles at sites of inflammation, including arthritic joints, ${ }^{25-27}$ has been suggested to be due to locally enhanced capillary permeability. ${ }^{28}$ The protective effect of DAPT against inflammatory arthritis was confirmed by in vivo non-invasive near-infrared fluorescence (NIRF) imaging, in which NIRF dye (Cy5.5-labelled HGC) nanoparticles were used to evaluate CIA induction. ${ }^{29}$ The increased and specific uptake of fluorescent HGC nanoparticles in arthritic tissues results from an enhanced permeation and retention effect. ${ }^{30-32}$ While
A

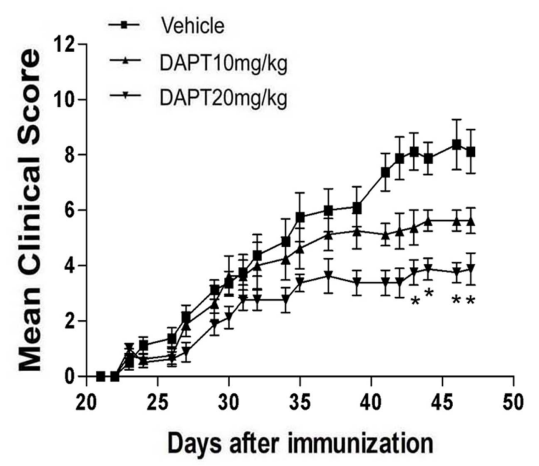

B

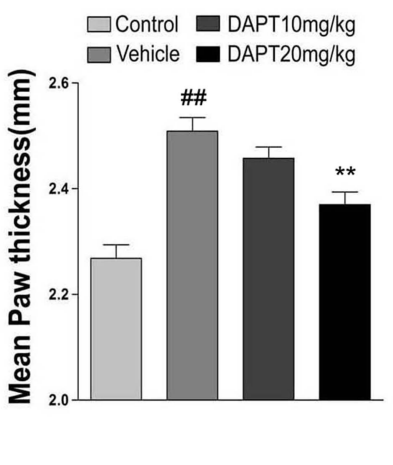

C

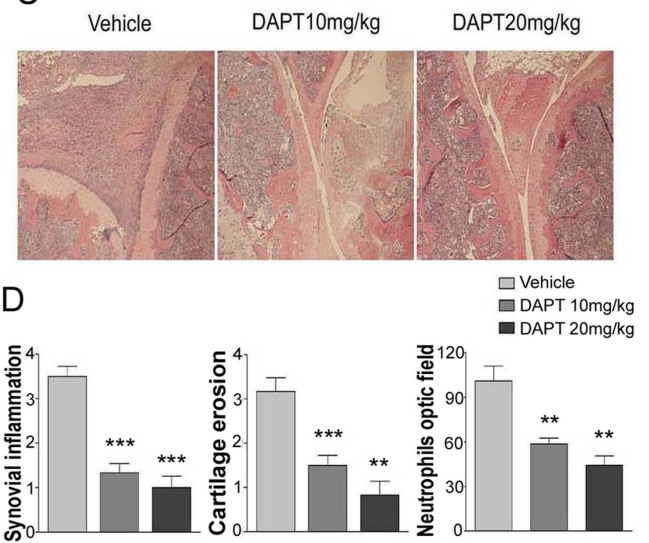

Figure 1 A $\gamma$-secretase inhibitor ameliorates symptoms of collagen-induced arthritis $(C I A)$ and inhibits in vivo biological responses. (A, B) The severity of arthritis was assessed using a visual arthritis scoring system (A) and calliper measurements (B) of paw thicknesses in CIA mice treated with vehicle or DAPT. Values are means \pm SEM $(n=8)$. \#\#p $<0.01$ versus control; ${ }^{*} p<0.01$ versus vehicle. (C) Representative sections of the knee joints of CIA mice 42 days after primary immunisation. Paw sections were histologically evaluated after H\&E staining. Original magnification, 200x. (D) Histological scores of synovial inflammation, cartilage erosion and neutrophil infiltration. Values are means $\pm S E M(n=4)$. ${ }^{* *} p<0.01,{ }^{* * *} p<0.001$. 
A

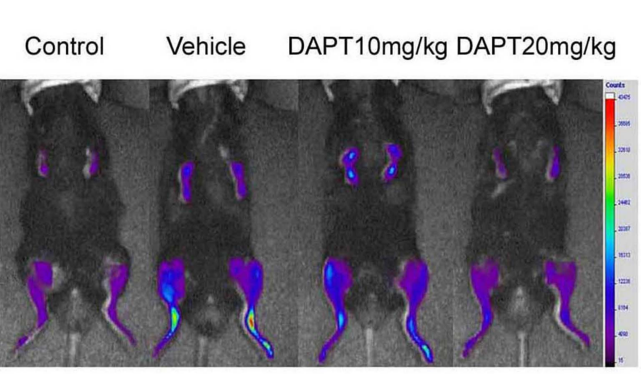

B

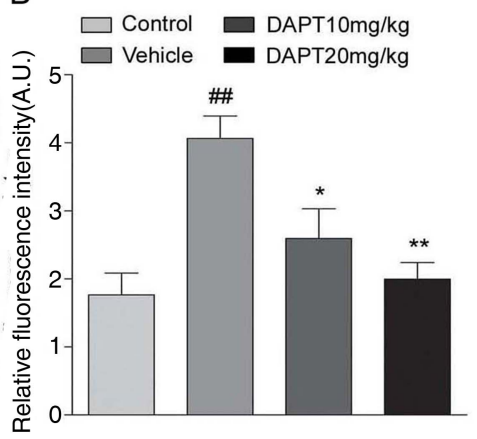

C

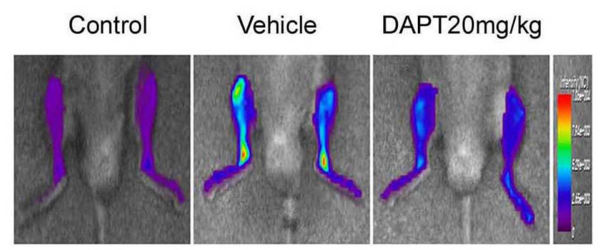

D $\square$ Control

$\square$ Vehicle $\left.\Im^{6}\right\rceil \square$ DAPT20mg/kg

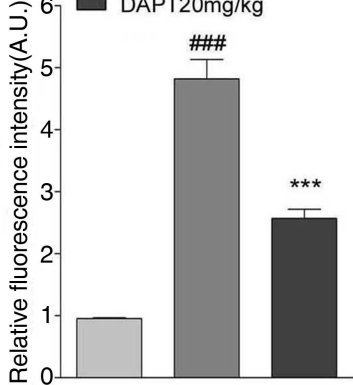

E

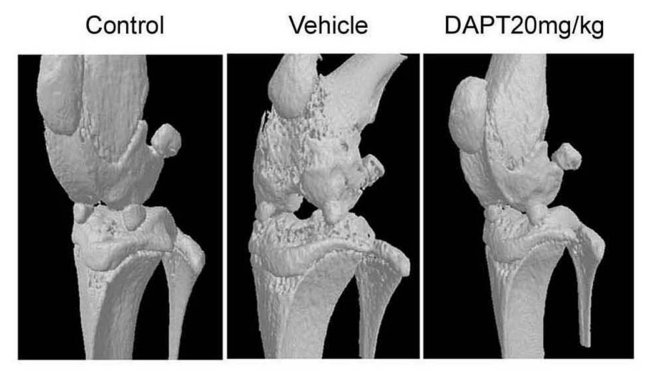

Figure 2 In vivo imaging and quantitative analysis of the progression of rheumatoid arthritis (RA), and its modification by $\gamma$-secretase inhibition, in collagen-induced arthritis (CIA) mice. (A) In vivo non-invasive near-infrared fluorescence (NIRF) images of CIA mice after intravenous injection of fluorescent hydrophobically modified glycol chitosan (HGC) nanoparticles at 45 days after primary immunisation. (B) Quantitative analysis showed a significant reduction in fluorescence intensity of HGC nanoparticles in DAPT-treated CIA mice. Values are means \pm SEM $(n=3)$. \#\#p $<0.01$ versus control; ${ }^{*} p<0.05,{ }^{* *} p<0.01$ versus vehicle. (C) Evaluation of inflammatory arthritis in CIA mice using a fluorogenic matrix metalloproteinase-3 (MMP-3)-specific polymeric probe. Representative in vivo NIRF tomographic images of a CIA mouse after intravenously injection of the MMP-3-specific polymeric probe on 30 days following first CII injection. (D) Quantitative analysis of fluorescence intensity in DAPT-treated CIA mice. Values are means $\pm S E M(n=3)$. \#\#\#p $<0.001$ versus control, ${ }^{* * *} p<0.001$ versus vehicle. (E) Representative three-dimensional reconstructions of micro-CT images of joint tissues and the hind limbs of collagen-naive, CIA and DAPT-treated CIA mice at 35 days after the first CII immunisation.

NIRF fluorescence was clearly detected in the arthritic joints of CIA mice, it was significantly reduced in DAPT-treated CIA mice (figure 2A,B). Matrix metalloproteinase-3 (MMP-3) is thought to be particularly important in the pathogenesis of RA. $^{33} 34$ The MMP-3-specific polymeric probe was developed by conjugating MMP-3-specific peptide probes, consisting of NIRF dye (FPR-675), MMP substrate peptide and dark quencher (BHQ-3), to self-assembled HGC nanoparticles; this probe enables a clear early diagnosis and visualisation of the arthritis progression using an NIRF imaging system. ${ }^{35}$ Next, we examined MMP-3 activity in CIA mice. When a MMP-3-specific polymeric probe was applied to the CIA mice, NIRF signal intensity also significantly decreased in the joints of DAPT-treated CIA mice indicating decreased MMP-3 activity (figure 2C,D). Moreover, in reconstructed three-dimensional micro-CT images of the joint tissues and hind limbs, loss of bone integrity and damage were found to be reduced in CIA mice by the administration of DAPT (figure 2E). These results verify that the $\gamma$-secretase inhibitor DAPT has protective effects against the propagation of arthritis in the CIA mice.

\section{Involvement of Notch-mediated nuclear factor (NF)-KB signalling in inflammatory arthritis in CIA mice}

Next, we measured the activity of $\gamma$-secretase and the levels of Notch, NICD and Notch downstream genes in joint samples from mice that had been subjected to CIA. Increased level of $\gamma$-secretase, activity in the joints of CIA mice was dosedependently decreased in the joints of mice treated with DAPT (figure 3A). We also found significant increase in Notch, NICD and Hes-1 (a gene downstream of Notch) protein levels in the joints of CIA mice versus collagen-naïve controls (figure 3B,C). The latter alterations were significantly suppressed in the joints of mice treated with DAPT (figure 3B,C). In addition, there were substantial increases in the expression of intercellular adhesion molecule 1 (ICAM-1) and active NF- $\mathrm{KB}$ in inflamed joints of CIA mice (figure 3B,C). The levels of ICAM-1 and active NF- $\mathrm{KB}$ were significantly suppressed in joints of DAPT-treated CIA mice (figure $3 \mathrm{~B}, \mathrm{C}$ ). We also confirmed these results using synoviocytes isolated from RA patients. Hes-1 and ICAM-1 mRNA expression were significantly reduced by treatments with the selective $\gamma$-secretase inhibitors including DAPT and L-685,458 in RA synovial fibroblasts (figure 3D).

In addition, we determined the levels of Notch ligands Jagged-1 and DLL-4 in joint samples from mice that had been subjected to CIA. We found significant increases in both Jagged-1 and DLL-4 levels in the joints of CIA mice (see online supplementary figure S1). However, these increments were not affected by DAPT treatments. Lipopolysaccharide (LPS) stimulation increased the levels of Jagged-1 and DLL-4 in RA synoviocytes, but this effect was not inhibited by $\gamma$-secretase inhibitors (see online supplementary figure S1).

\section{$\gamma$-secretase inhibitors reduce the production of proinflammatory mediators in CIA mice and RA synovial fibroblasts}

All proinflammatory cytokines examined (TNF- $\alpha$, IFN- $\gamma$, IL-6, IL-12, IL-17 and MCP-1), with the exception of the antiinflammatory cytokine IL-10, were significantly elevated in CIA 
A

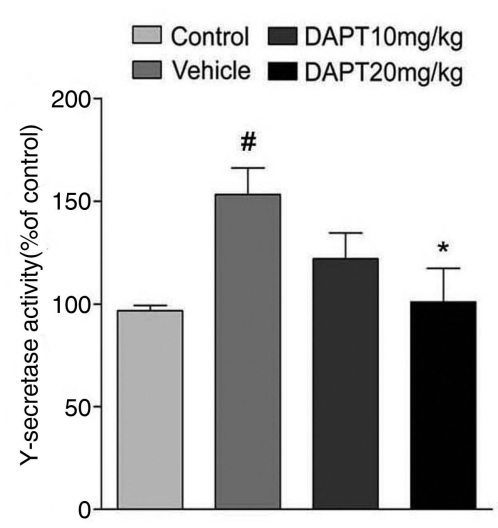

B

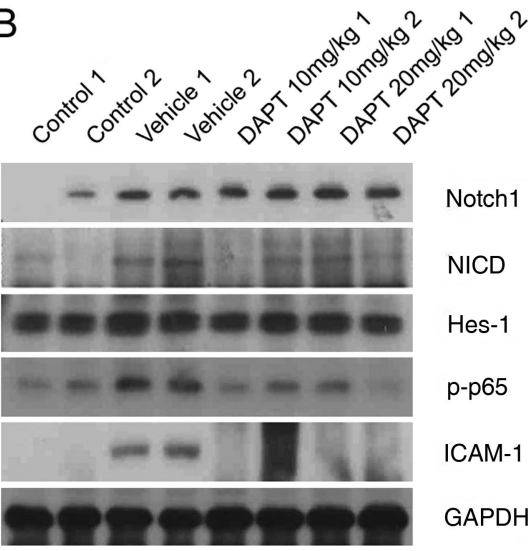

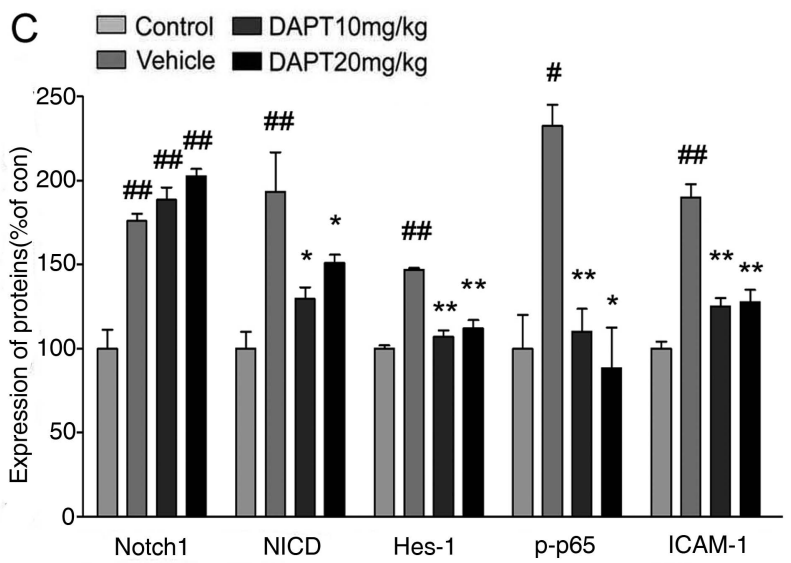

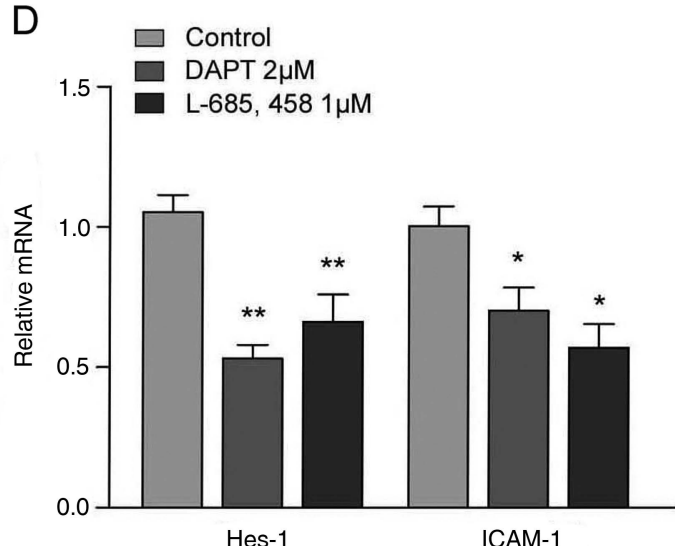

Figure 3 Notch signalling mediates NF- $\kappa B$ signalling and inflammatory processes in experimental models of rheumatoid arthritis (RA). (A) $\gamma$-secretase activity in the knee joints of control mice and of collagen-induced arthritis (CIA) mice treated with vehicle or DAPT at 42 days after primary immunisation of $\mathrm{Cll}$, was determined. Values are means \pm SEM $(n=5)$. $\# p<0.05$ versus control; ${ }^{*} p<0.05$ versus vehicle. (B) Proteins in joint tissue from control or CIA mice treated with vehicle or DAPT were immunoblotted using antibodies against Notch-1, Notch intracellular domain (NICD), Hes-1, ICAM-1, phospho-p65 and GAPDH. Each lane is a sample from a different mouse. (C) Protein levels in immunoblots were normalised versus GAPDH levels. Values are means $\pm \operatorname{SEM}(n=3), \# p<0.05, \# \# p<0.01 ;{ }^{*} p<0.05,{ }^{*} p<0.01$ versus vehicle. (D) Relative mRNA expression of Hes-1 and ICAM-1 was assessed by quantitative real-time PCR after $2 \mu \mathrm{M}$ DAPT or $1 \mu \mathrm{M}$ L-685,458 treatment for $24 \mathrm{~h}$ in synoviocytes of rheumatoid arthritis (RA) patients. Values are means \pm SEM $(n=6)$. ${ }^{*} p<0.05,{ }^{* *} p<0.01$.

mouse serum, and DAPT significantly suppressed the levels of these cytokines (figure 4A). In addition, DAPT or L-685,458 treatment substantially decreased mRNA expression of proinflammatory cytokines (TNF- $\alpha$, IL-6, IL-12, IL-17 and MCP-1) in RA synoviocytes isolated from RA patients, whereas IL-10 expression was not affected by $\gamma$-secretase inhibitors (figure 4B). We also confirmed that the protein levels of MMP-3, TNF- $\alpha$ and IL-6, key players in the pathogenesis of RA, were elevated in LPS-stimulated RA synoviocytes. DAPT or L-685,458 treatment significantly decreased MMP-3, TNF- $\alpha$ and IL- 6 production in LPS-activated RA synoviocytes (figure 4C-F). Consistently, the productions of TNF- $\alpha$ and IFN- $\gamma$ by splenocytes were stimulated by Jagged-1 (a Notch ligand) and the inductions of TNF- $\alpha$ and IFN- $\gamma$ were blocked by DAPT (see online supplementary figure S2). Furthermore, the administration of DAPT after the onset of CIA was also effective in reducing the production of pathogenic antitype II collagen IgG1 and IgG2a (see online supplementary figure S3).

\section{$\mathrm{CIA}$ progression is attenuated in NAS transgenic mice}

Encouraged by the efficacy of $\gamma$-secretase inhibitors in our rodent model of RA, we examined mice transgenic for antisense Notch $(\mathrm{NAS})^{20}$ with CIA to determine whether a specific reduction of Notch signalling attenuates the severity of inflammatory arthritis. NAS transgenic mice express 30-40\% less Notch protein than do wild-type (WT) mice. ${ }^{20}$ Arthritis indices, paw thicknesses, synovitis, cartilage erosion and neutrophil infiltration resulting from CIA were significantly less in NAS mice compared with WT mice (figure 5A-D). The reduced inflammation of arthritic joints observed in NAS CIA mice was confirmed by in vivo NIRF imaging using Cy5.5-labelled HGC nanoparticles (figure $5 \mathrm{E}, \mathrm{F}$ ). Serum proinflammatory cytokine (TNF- $\alpha$, IFN- $\gamma$, MCP-1, IL-12 and IL-17) levels were lower in NAS CIA mice than in WT CIA (figure $5 \mathrm{G}$ ).

\section{Notch inhibition ameliorates established arthritis}

We next evaluated whether Notch inhibition has therapeutic effects when $\gamma$-secretase inhibitor treatment is initiated after the disease is established in the CIA model. Measurable signs of arthritis typically developed 1-7 days after the secondary immunisation. After secondary immunisation, vehicle-treated DBA1/J mice developed severe paw swelling and joint inflammation with cartilage erosion and neutrophil infiltration (figure 6A-D). In therapeutic studies, when treatment started 11 days after secondary collagen immunisation (day 32), DAPT or L-685,458 significantly reversed CIA compared with vehicle 

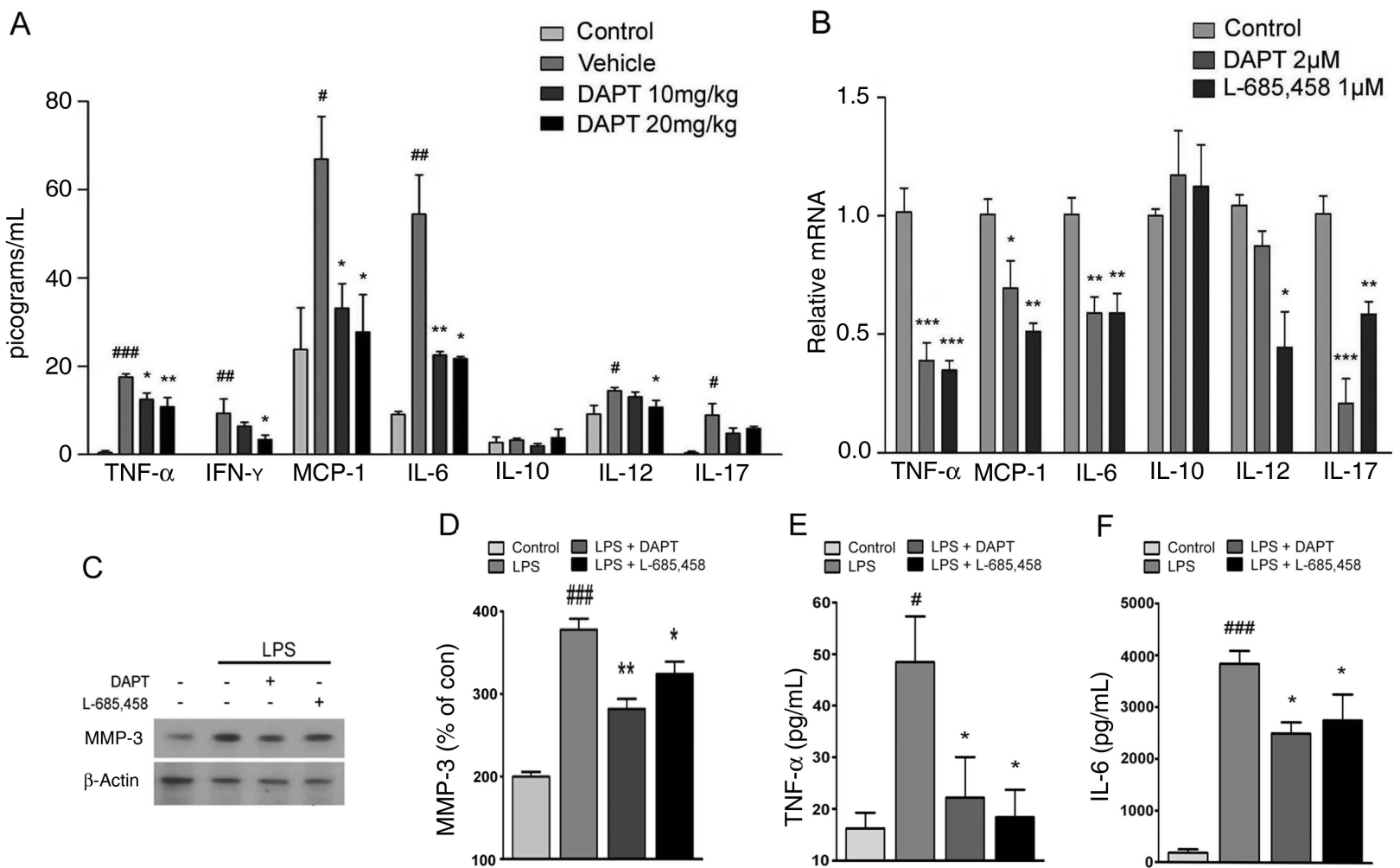

Figure 4 Suppression of proinflammatory cytokines by $\gamma$-secretase inhibitors in collagen-induced arthritis (CIA) mice and rheumatoid arthritis (RA) synoviocytes. (A) Bead-based cytokine analysis of serum using the Luminex 100 system, 49 days after primary immunisation in CIA mice. Values are means \pm SEM $(n=6)$. \#p $<0.05, \# \# p<0.01$, \#\#\#p<0.001 versus control; ${ }^{*} p<0.05,{ }^{* *} p<0.01$ versus vehicle. (B) Relative mRNA expression of cytokines was analysed by quantitative real-time PCR after synoviocytes obtained from RA patients were treated with $2 \mathrm{mM}$ DAPT or 1 mM L-685,458 for $24 \mathrm{~h}$. Values are means \pm SEM $(n=6) .{ }^{*} p<0.05,{ }^{*} p<0.01,{ }^{* * *} p<0.001$. (C, D) RA synoviocytes were pretreated with DAPT $(2 \mu M)$ or L-685,458 $(1 \mu \mathrm{M})$ for $2 \mathrm{~h}$. The cells were then exposed to LPS $(2 \mu \mathrm{g} / \mathrm{ml})$ for $24 \mathrm{~h}$. Matrix metalloproteinase-3 (MMP-3) levels were determined by western blots. Values are means \pm SEM $(n=3) \# \# \# p<0.001$ versus control; ${ }^{*} p<0.05,{ }^{* *} p<0.01$ versus LPS. ( $\left.E_{1}\right)$ Culture media were collected and analysed for TNF- $\alpha(E)$ and IL-6 (F) by ELISA. Values are means \pm SEM $(n=3)$. \#p $<0.05, \# \# p<0.001$ versus control; * $p<0.05$ versus LPS.

treatment (figure 6A,B). Treatment with DAPT or L-685,458 inhibited synovial inflammation, cartilage erosion and neutrophil infiltration in mice with established disease (figure 6C,D).

\section{DISCUSSION}

RA is an autoimmune disease characterised by chronic inflammation of joints and progressive destruction of cartilage and bone. Despite extensive efforts, the molecular pathogenesis and aetiology of RA is not yet fully understood, and effective treatments with limited side effects are lacking. Here we demonstrate that Notch signalling stimulates synoviocytes and accelerates their production of proinflammatory cytokines and immune responses involving the upregulation of IgG1 and IgG2a. Prophylactic or therapeutic inhibition of $\gamma$-secretase, an enzyme required for Notch activation, and antisense-mediated knockdown of Notch attenuates the severity of inflammatory arthritis, including arthritis indices, paw thickness, tissue damage and neutrophil infiltration, reduced the levels of active NF-кB, ICAM-1 and proinflammatory cytokines in the CIA model. These results suggest that Notch is involved in the pathogenesis of RA and that inhibition of Notch signalling is an approach for treating RA.

$\gamma$-secretase is the enzyme complex responsible for cleavage of Notch to release its active intracellular transactivator NICD. Several $\gamma$-secretase inhibitors have been widely used to analyse and examine Notch signalling. However, most of these inhibitors are not specific for $\gamma$-secretase cleavage of Notch, and equally inhibit the processing of many other $\gamma$-secretase substrates. ${ }^{36}$ Therefore, we examined NAS transgenic mice in which multiple cell types, including lymphocytes, exhibit $30-40 \%$ reduced levels of Notch protein. ${ }^{20}$ We found that interference with Notch expression reduced arthritis development as well as neutrophil accumulation in the joints in the CIA model.

$\gamma$-secretase-mediated activation of the Notch pathway is required for the full expression of NF- $\mathrm{KB}$ activation and production of INF- $\gamma$ in lymphocytes, suggesting that the crosstalk between these signalling pathways participates in modulating the responsiveness of immune cells to stimulation. ${ }^{37}{ }^{38}$ Notch signalling can also enhance $\mathrm{T}$ cell proliferation and cytokine production. ${ }^{39}$ Furthermore, the proinflammatory cytokines, IFN- $\gamma$, TNF- $\alpha$, IL- 6 , IL-12 and IL-17, are significant regulators of the pathophysiology of autoimmune diseases, including RA. Several previous studies have provided evidence that Notch signalling is involved in the pathophysiology of RA. Notch is activated in synovial membrane and synoviocytes from RA patients, and Notch can promote TNF $\alpha$-induced synoviocyte proliferation in vitro. $^{8-10}$ The Notch pathway also mediates VEGF/ Ang2-induced angiogenesis in inflammatory arthritis. ${ }^{13}{ }^{14}$ Consistent with the findings of those studies, we have shown that Notch signalling stimulates synoviocytes in vivo and accelerates their production of proinflammatory cytokines and immune responses involving the upregulation of $\operatorname{IgG} 1$ and $\operatorname{IgG} 2 \mathrm{a}$. Genetic inhibition and treatment with the pharmacological agents showed that blockage of Notch signalling reduced the 
A

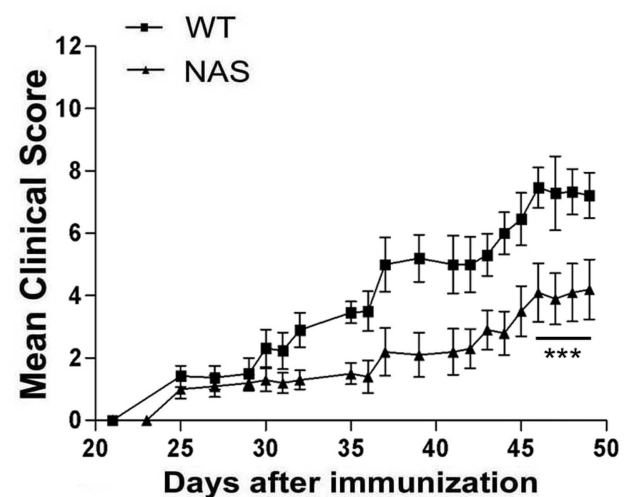

B

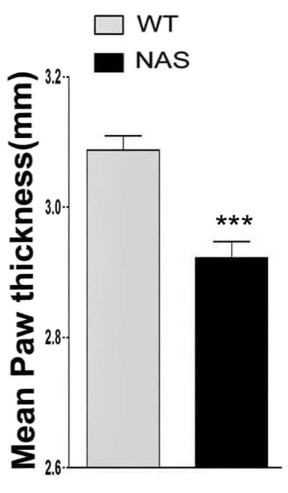

E

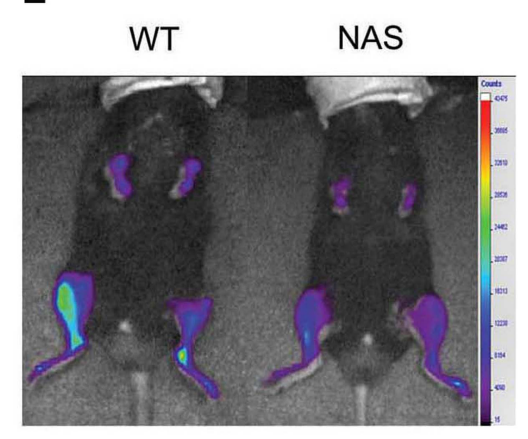

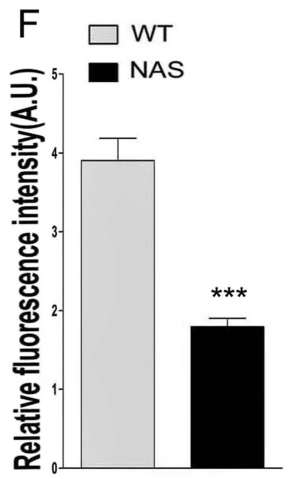

C<smiles>[Mg][Mg]</smiles>
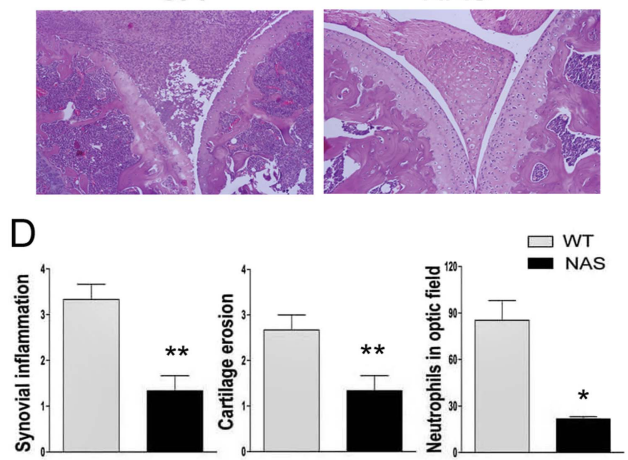

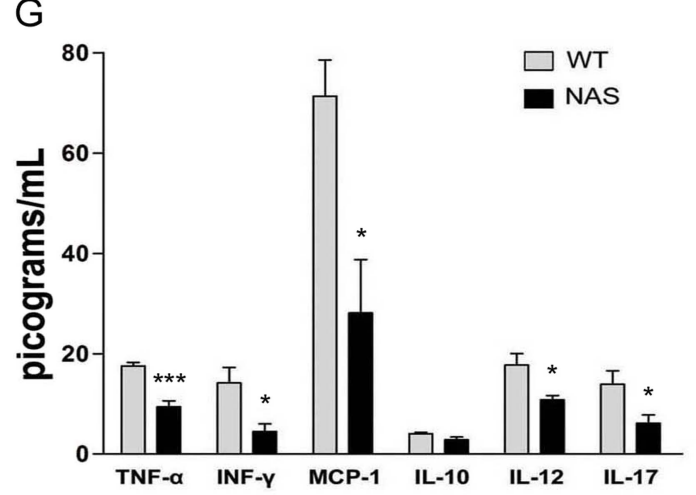

Figure 5 Collagen-induced arthritis (CIA) progression is reduced in Notch-1-antisense (NAS) transgenic mice. (A, B) Severities of arthritis (A) and paw thickness measurements (B) in NAS or non-transgenic (wild-type (WT)) CIA mice. Values are means $\pm S E M(n=8) .{ }^{* *} p<0.001$. (C) Representative photomicrographs of H\&E-stained tissue sections of knee joints of WT and NAS CIA mice at 49 days after primary immunisation. Original magnification, 200x. (D) Quantification of the histological findings of synovitis, erosion of bone and cartilage, and neutrophil infiltration. Values are means \pm SEM $(n=4) .{ }^{*} p<0.05,{ }^{*} p<0.01$. (E) In vivo non-invasive near-infrared fluorescence (NIRF) images of NAS or WT CIA mice. (F) Quantitative analysis of fluorescence intensities in the joints of NAS CIA mice. Values are means \pm SEM $(n=3)$. ${ }^{* *} p<0.001$. (G) Bead-based cytokine serum analysis at 49 days after primary immunisation. Values are means \pm SEM $(n=8) .{ }^{*} p<0.05,{ }^{* * *} p<0.001$.

levels of active NF- $\mathrm{KB}$, ICAM-1 and proinflammatory cytokines in the CIA model. We also confirmed that $\gamma$-secretase inhibitors reduced the relative mRNA levels of Hes-1, ICAM-1 and proinflammatory cytokines in human RA synoviocytes. These results are consistent with previous reports on the relationship between Notch/Hes-1, NF- $\mathrm{B}$ and ICAM-1. ${ }^{37} 40$ Notch signalling can sustain NF- $\kappa$ B activation, ${ }^{20} 37$ which is essential for ICAM-1 expression, and the upregulation of ICAM-1 enhances the adhesion of leucocytes to synovial fibroblasts in RA. ${ }^{40}$ Collectively, these results suggest that $\gamma$-secretase inhibitors have suppressive effects against the propagation of arthritis by suppressing the $\gamma$-secretase-Notch-NF- $\kappa \mathrm{B}$ axis that induces the expression of ICAM-1 in the CIA mice and primary human RA synoviocytes.

We found that the expression of Jagged-1 and DLL-4 is increased in the inflamed joints and RA synoviocytes. It has also been shown that there is a high level of expression of DLL-1, Jagged-1, Notch-1, Notch-2 and Notch-3 in the synovium of RA patients. ${ }^{9}{ }^{41}$ However, the precise role of each Notch ligand in autoimmune diseases remains unclear. The inhibition of Notch signalling by a $\gamma$-secretase inhibitor suppressed the progression of experimental autoimmune encephalomyelitis (EAE). ${ }^{15}$ In contrast, overexpression of Jagged-1 suppressed CIA, and the blockade of Jagged-1 by an anti-Jagged-1 monoclonal antibody exacerbated CIA. ${ }^{11}$ Another report demonstrated that the administration of anti-Jagged-1 mAb exacerbated the clinical features of EAE, whereas DLL-1 mAb reduced disease severity. ${ }^{42}$ Similarly, DLL-1 promotes osteoclastogenesis, while Jagged-1 suppressed osteoclastogenesis. $^{43}$ Also, it has been suggested that the Notch ligands Jagged-1 and DLL-4 have distinct spatial expression pattern and opposing effects on angiogenesis. ${ }^{44}$ In addition, Notch is a positive regulator of DLL-4 but not of Jagged-1, while inflammatory factors such as TNF $\alpha$ induce Jagged-1 but decrease DLL-4 expression. ${ }^{44} 46-48$ These data suggest complex contextdependent roles of Notch ligands in regulating inflammation and angiogenesis, and suggest the possibility that an endogenous ligand other than Jagged-1 mediates the adverse effects of Notch signalling in the CIA mouse model.

Our findings suggest that inhibition of Notch signalling in immune cells of inflamed joints may have a therapeutic benefit in RA. However, treatments that specifically target Notch in the subpopulation of lymphocytes activated in RA will likely be required to avoid unwanted side effects of $\gamma$-secretase inhibitors that block cleavage of Notch in all cells, and also block cleavage of other $\gamma$-secretase substrates. Similarly, agents that block interaction of some Notch ligands with Notch would be expected to inhibit the proliferation of stem cells in many different tissues. ${ }^{49}$ Nevertheless, our findings suggest that even global inhibition of Notch signalling markedly reduces the clinical signs of RA and immune cell-mediated damage to joint tissue in an animal model. Because the current treatments for RA do not prevent the damage to joint tissues, our findings suggest that further studies of treatment strategies that target Notch signalling in RA are warranted. 

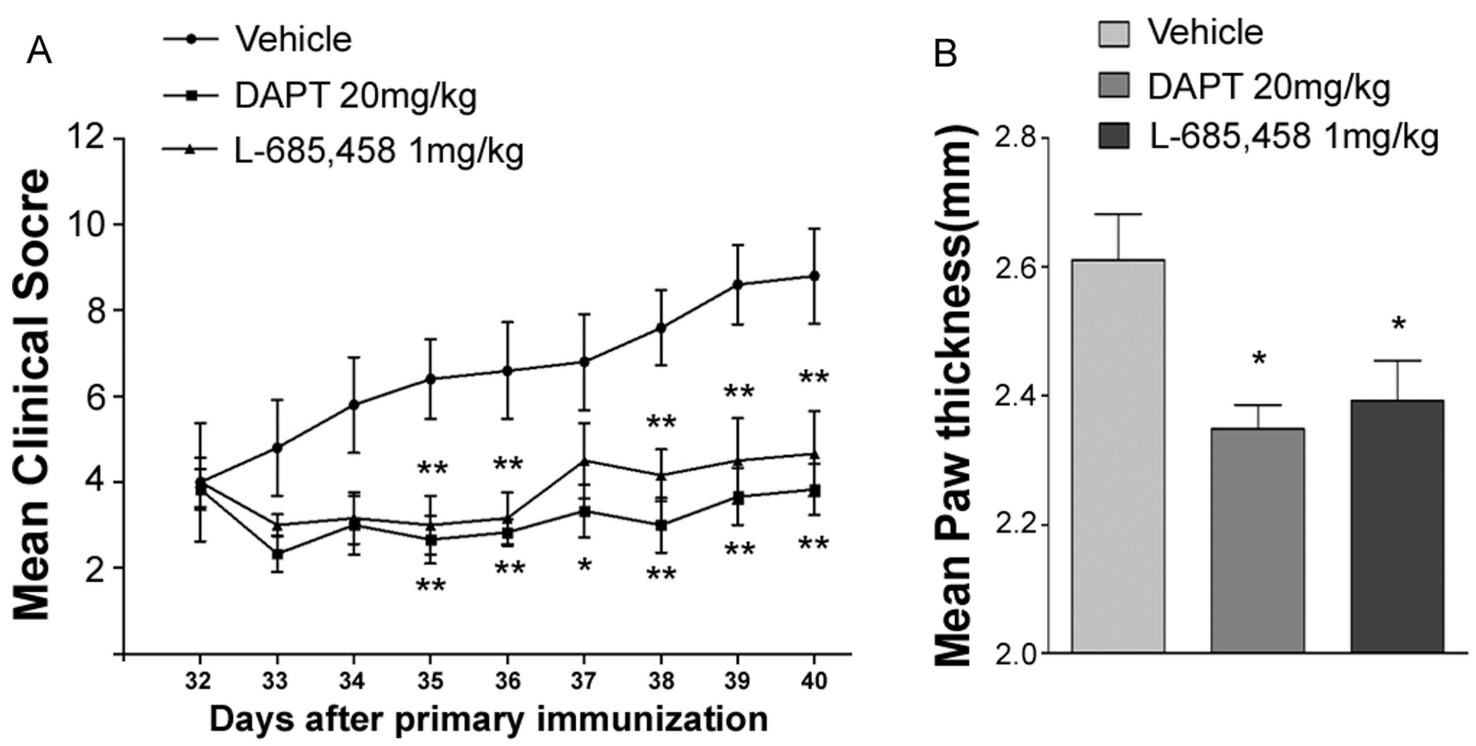

C

Vehicle

DAPT $20 \mathrm{mg} / \mathrm{kg}$

$\mathrm{L}-685,458$ 1mg/kg
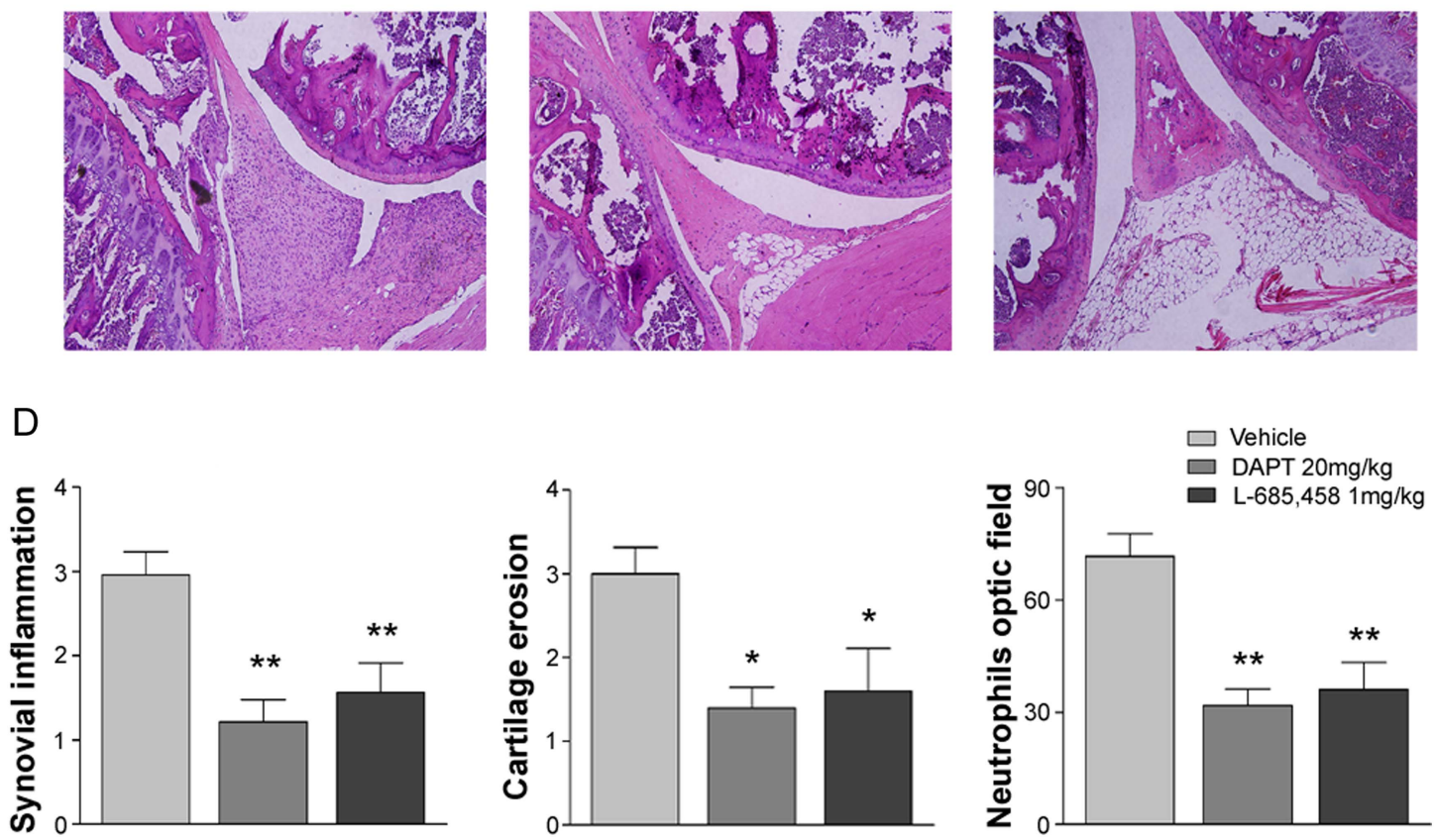

Figure 6 Therapeutic efficacy of $\gamma$-secretase inhibitors in collagen-induced arthritis (CIA). (A) CIA mice were administered DAPT or L-685,458 starting at 32 days after primary immunisation. Severities of arthritis were assessed using a visual arthritis scoring system (A) and calliper measurements (B) of paw thicknesses in CIA mice treated with vehicle, DAPT or L-685,458. Values are means \pm SEM $(n=5)$. ${ }^{*} p<0.05,{ }^{* *} p<0.01$. (C) Representative photomicrographs of H\&E-stained tissue sections of the knee joints of control mice and CIA mice treated with vehicle, DAPT, or L-685,458. Original magnification, 200x. (D) Quantification of synovial inflammation, cartilage erosion and neutrophil infiltration. Values are means \pm SEM $(n=3) .{ }^{*} p<0.05,{ }^{* *} p<0.01$.

\section{Author affiliations}

${ }^{1}$ School of Pharmacy, Sungkyunkwan University, Suwon, Korea

${ }^{2}$ Departments of Polymer Science and Chemical Engineering, Sungkyunkwan University, Suwon, Korea

${ }^{3}$ Biomedical Research Center, Korea Institute of Science and Technology, Seoul, Korea

${ }^{4}$ Department of Biochemistry and Molecular Biology, College of Life Science \& Technology, Heilongjiang Bayi Agricultural University, Daqing, China

${ }^{5}$ Laboratory of Molecular Imaging and Nanomedicine, National Institute of Biomedical Imaging and Bioengineering, National Institutes of Health, Bethesda, Maryland, USA

${ }^{6}$ Russell H. Morgan Department of Radiology and Radiological Science, Center for Cancer Nanotechnology Excellence, Center for Nanomedicine at the Wilmer Eye Institute, Johns Hopkins University, Baltimore, Maryland, USA
${ }^{7}$ Department of Internal Medicine (Rheumatology), Kyungpook National University School of Medicine, Daegu, Korea

${ }^{8}$ Department of Physiology, Yong Loo Lin School Medicine, National University of Singapore, Singapore, Singapore

${ }^{9}$ Laboratory of Neurosciences, National Institute on Aging Intramural Research Program, Baltimore, Maryland, USA

${ }^{10}$ Department of Neuroscience, Johns Hopkins University School of Medicine, Baltimore, Maryland, USA

Acknowledgements This research was supported by the National Research Foundation of Korea grants (2009-0069827, 2012-0009851,

2012R1A2A2A01047551), a grant of the Korea Healthcare Technology R\&D project (A092042) from the Korean Government and the Intramural Research Program of the National Institute on Aging, NIH. 
Contributors JSP, SHK, KK, JHP and DGJ designed the project. JSP, SHK, CHJ, $K Y C, J J, Y C, A R G$, SHB, UY and YC performed research. KK, SYC, SL, YMK, KCL, TVA and MPM contributed new reagents/analytic tools. JSP, SHK, KK, JHP and DGJ analysed data. JSP, SHK, KK, MPM, JHP and DGJ wrote the manuscript.

Funding The Korea Healthcare Technology R\&D project (A092042) and National Research Foundation of Korean Government (2012-0009851,

2012R1A2A2A01047551, 2012R1A1A2009093).

Competing interests None.

Patient consent Obtained.

Provenance and peer review Not commissioned; externally peer reviewed.

\section{REFERENCES}

1 Fiuza UM, Arias AM. Cell and molecular biology of Notch. J Endocrinol 2007;194:459-74.

2 Bray S, Bernard F. Notch targets and their regulation. Curr Top Dev Biol 2010;92:253-75.

3 Selkoe D, Kopan R. Notch and Presenilin: regulated intramembrane proteolysis links development and degeneration. Annu Rev Neurosci 2003;26:565-97.

4 Woo HN, Park JS, Gwon AR, et al. Alzheimer's disease and Notch signaling. Biochem Biophys Res Commun 2009;390:1093-7.

5 McInnes IB, Schett G. Cytokines in the pathogenesis of rheumatoid arthritis. Nat Rev Immunol 2007;7:429-42.

6 Feldmann M, Brennan FM, Maini RN. Role of cytokines in rheumatoid arthritis. Annu Rev Immunol 1996;14:397-440.

7 Khan IM, Palmer EA, Archer CW. Fibroblast growth factor-2 induced chondrocyte cluster formation in experimentally wounded articular cartilage is blocked by soluble Jagged-1. Osteoarthritis Cartilage 2010;18:208-19.

8 Nakazawa M, Ishii $\mathrm{H}$, Aono $\mathrm{H}$, et al. Role of Notch-1 intracellular domain in activation of rheumatoid synoviocytes. Arthritis Rheum 2001;44:1545-54.

9 Ishii H, Nakazawa M, Yoshino S, et al. Expression of notch homologues in the synovium of rheumatoid arthritis and osteoarthritis patients. Rheumatol Int 2001;21:10-14.

10 Ando K, Kanazawa S, Tetsuka T, et al. Induction of Notch signaling by tumor necrosis factor in rheumatoid synovial fibroblasts. Oncogene 2003;22:7796-803.

11 Kijima M, Iwata A, Maekawa Y, et al. Jagged1 suppresses collagen-induced arthritis by indirectly providing a negative signal in CD8+ T cells. J Immunol 2009;182:3566-72.

12 Jiao Z, Wang W, Guo M, et al. Expression analysis of Notch-related molecules in peripheral blood Thelper cells of patients with rheumatoid arthritis. Scand J Rheumatol 2010;39:26-32.

13 Gao W, Sweeney C, Connolly M, et al. Notch-1 mediates hypoxia-induced angiogenesis in rheumatoid arthritis. Arthritis Rheum 2012;64:2104-13.

14 Gao W, Sweeney C, Walsh C, et al. Notch signalling pathways mediate synovial angiogenesis in response to vascular endothelial growth factor and angiopoietin 2. Ann Rheum Dis 2013;72:1080-8.

15 Minter LM, Turley DM, Das $\mathrm{P}$, et al. Inhibitors of gamma-secretase block in vivo and in vitro $T$ helper type 1 polarization by preventing Notch upregulation of Tbx21. Nat Immunol 2005;6:680-8.

16 Niranjan T, Bielesz B, Gruenwald A, et al. The Notch pathway in podocytes plays a role in the development of glomerular disease. Nat Med 2008;14:290-8.

17 Arumugam TV, Chan SL, Jo DG, et al. Gamma secretase-mediated Notch signaling worsens brain damage and functional outcome in ischemic stroke. Nat Med 2006;12:621-3.

18 Arumugam TV, Cheng YL, Choi Y, et al. Evidence that gamma-secretase-mediated Notch signaling induces neuronal cell death via the nuclear factor-kappaB-BCl-2interacting mediator of cell death pathway in ischemic stroke. Mol Pharmacol 2011;80:23-31.

19 Wei Z, Chigurupati S, Arumugam TV, et al. Notch activation enhances the microglia-mediated inflammatory response associated with focal cerebral ischemia. Stroke 2011;42:2589-94.

20 Cheng P, Zlobin A, Volgina V, et al. Notch-1 regulates NF-kappaB activity in hemopoietic progenitor cells. J Immunol 2001;167:4458-67.

21 Inglis JJ, Simelyte E, McCann FE, et al. Protocol for the induction of arthritis in C57BL/6 mice. Nat Protoc 2008;3:612-18.

22 Arnett FC, Edworthy SM, Bloch DA, et al. The American Rheumatism Association 1987 revised criteria for the classification of rheumatoid arthritis. Arthritis Rheum $1988 ; 31: 315-24$
23 Park JH, Kwon S, Nam JO, et al. Self-assembled nanoparticles based on glycol chitosan bearing 5beta-cholanic acid for RGD peptide delivery. I Control Release 2004;95:579-88.

24 Feldmann M, Maini RN. Lasker Clinical Medical Research Award. TNF defined as a therapeutic target for rheumatoid arthritis and other autoimmune diseases. Nat Med 2003;9:1245-50.

25 Metselaar JM, Wauben MH, Wagenaar-Hilbers JP, et al. Complete remission of experimental arthritis by joint targeting of glucocorticoids with long-circulating liposomes. Arthritis Rheum 2003;48:2059-66.

26 Boerman OC, Oyen WJ, Storm G, et al. Technetium-99 m labelled liposomes to image experimental arthritis. Ann Rheum Dis 1997;56:369-73.

27 Dams ET, Reijnen MM, Oyen WJ, et al. Imaging experimental intraabdominal abscesses with 99mTc-PEG liposomes and 99mTc-HYNIC IgG. Ann Surg 1999;229:551-7.

28 Paleolog EM, Fava RA. Angiogenesis in rheumatoid arthritis: implications for future therapeutic strategies. Springer Semin Immunopathol 1998;20:73-94.

29 Park K, Kim JH, Nam YS, et al. Effect of polymer molecular weight on the tumor targeting characteristics of self-assembled glycol chitosan nanoparticles. I Control Release 2007:122:305-14.

$30 \mathrm{Koo} \mathrm{H}$, Huh MS, Sun IC, et al. In Vivo Targeted Delivery of Nanoparticles for Theranosis. Acc Chem Res 2011;44:1018-28.

31 Lee S, Cha EJ, Park K, et al. A near-infrared-fluorescence-quenched gold-nanoparticle imaging probe for in vivo drug screening and protease activity determination. Angew Chem Int Ed Engl 2008;47:2804-7.

32 Sandanaraj BS, Gremlich HU, Kneuer R, et al. Fluorescent nanoprobes as a biomarker for increased vascular permeability: implications in diagnosis and treatment of cancer and inflammation. Bioconjug Chem 2010;21:93-101.

33 Kevorkian L, Young DA, Darrah C, et al. Expression profiling of metalloproteinases and their inhibitors in cartilage. Arthritis Rheum 2004;50:131-41.

34 Eguchi T, Kubota S, Kawata K, et al. Novel transcription-factor-like function of human matrix metalloproteinase 3 regulating the CTGF/CCN2 gene. Mol Cell Biol 2008:28:2391-413.

35 Ryu JH, Lee A, Chu JU, et al. Early diagnosis of arthritis in mice with collagen-induced arthritis, using a fluorogenic matrix metalloproteinase 3-specific polymeric probe. Arthritis Rheum 2011;63:3824-32.

36 Woo HN, Baik SH, Park JS, et al. Secretases as therapeutic targets for Alzheimer's disease. Biochem Biophys Res Commun 2011;404:10-5.

37 Espinosa L, Cathelin S, D'Altri T, et al. The Notch/Hes1 pathway sustains NF-kappaB activation through CYLD repression in T cell leukemia. Cancer Cell 2010;18:268-81

38 Shin HM, Minter LM, Cho OH, et al. Notch1 augments NF-kappaB activity by facilitating its nuclear retention. EMBO J 2006;25:129-38.

39 Maillard I, Fang T, Pear WS. Regulation of lymphoid development, differentiation, and function by the Notch pathway. Annu Rev Immunol 2005;23:945-74.

40 Yang CM, Luo SF, Hsieh HL, et al. Interleukin-1 beta induces ICAM-1 expression enhancing leukocyte adhesion in human rheumatoid arthritis synovial fibroblasts: involvement of ERK, JNK, AP-1, and NF-kappaB. J Cell Physiol 2010;224:516-26.

41 Yabe Y, Matsumoto T, Tsurumoto T, et al. Immunohistological localization of Notch receptors and their ligands Delta and Jagged in synovial tissues of rheumatoid arthritis. J Orthop Sci 2005;10:589-94.

42 Elyaman W, Bradshaw EM, Wang Y, et al. JAGGED1 and delta1 differentially regulate the outcome of experimental autoimmune encephalomyelitis. I Immunol 2007; 179:5990-8.

43 Sekine C, Koyanagi A, Koyama N, et al. Differential regulation of osteoclastogenesis by Notch2/Delta-like 1 and Notch1/Jagged1 axes. Arthritis Res Ther 2012;14:R45.

44 Benedito R, Roca C, Sorensen I, et al. The notch ligands DII4 and Jagged1 have opposing effects on angiogenesis. Cell 2009;137:1124-35.

45 Hellstrom M, Phng LK, Hofmann JJ, et al. DII4 signalling through Notch1 regulates formation of tip cells during angiogenesis. Nature 2007;445:776-80.

46 Ridgway J, Zhang G, Wu Y, et al. Inhibition of DII4 signalling inhibits tumour growth by deregulating angiogenesis. Nature 2006;444:1083-7.

47 Shawber CJ, Das I, Francisco E, et al. Notch signaling in primary endothelial cells. Ann N Y Acad Sci 2003;995:162-70.

48 Sainson RC, Johnston DA, Chu HC, et al. TNF primes endothelial cells for angiogenic sprouting by inducing a tip cell phenotype. Blood 2008:111:4997-5007.

49 Radtke F, Wilson A, Mancini SJ, et al. Notch regulation of lymphocyte development and function. Nat Immunol 2004;5:247-53. 